\title{
Expression of BMI1 and ZEB1 in epithelial-mesenchymal transition of tongue squamous cell carcinoma
}

\author{
KINUE KURIHARA ${ }^{1}$, TOMOHIDE ISOBE ${ }^{2}$, GOU YAMAMOTO ${ }^{2}$, YOICHI TANAKA ${ }^{3}$, \\ AKIRA KATAKURA $^{1}$ and TETSUHIKO TACHIKAWA ${ }^{4}$
}

\author{
${ }^{1}$ Department of Oral Medicine, Oral and Maxillofacial Surgery, Tokyo Dental College, Ichikawa, Chiba 272-8513; \\ ${ }^{2}$ Division of Pathology, Department of Oral Diagnostic Sciences, School of Dentistry, Showa University, Shinagawa-ku, \\ Tokyo 142-8555; ${ }^{3}$ Division of Surgical Pathology, Clinical Laboratory, Ichikawa General Hospital, \\ Ichikawa, Chiba 272-8513; ${ }^{4}$ Innovative Research Center of Oral Cancer, \\ Showa University, Shinagawa-ku, Tokyo 142-8555, Japan
}

Received March 13, 2015; Accepted May 6, 2015

DOI: 10.3892/or.2015.4032

\begin{abstract}
Epithelial-mesenchymal transition (EMT) is a crucial event required for the invasion and progression of carcinogenesis, inducing stem-like properties in epithelial cells. In the present study, the expression of BMI1, which controls self-renewal in stem cells, as well as that of ZEB1, a transcription factor that regulates EMT, was evaluated for its role in EMT and the carcinogenic processes of tongue squamous cell carcinoma (TSCC). Collagen invasion assays using two TSCC cells and 64 tongue specimens (32 carcinomas and 32 dysplasias) were employed and analyzed in the present study. We assessed the protein and mRNA expression levels of BMI, ZEB1, vimentin and E-cadherin in the two cell lines and tumor tissues. The protein and mRNA expression of BMI1 and ZEB1 occurred at the invasion of TSCC. The elevated levels of BMI1 and ZEB1 were accompanied by the downregulation of E-cadherin and upregulation of vimentin at the invasive front, indicative of EMT in vitro and in vivo. The results showed that BMI1 and ZEB1 are important factors in association with the promotion of EMT and invasion of TSCC.
\end{abstract}

\section{Introduction}

Head and neck squamous cell carcinoma (HNSCC), including oral cancer, is the sixth most prevalent cancer worldwide and accounts for $\sim 8-10 \%$ of all cancer types in Southeast Asia $(1,2)$. Despite improvements in the diagnosis and management of HNSCC, long-term survival rates have improved only marginally over the past decade (3). To improve the survival rate in

Correspondence to: Dr Kinue Kurihara, Department of Oral Medicine, Oral and Maxillofacial Surgery, Tokyo Dental College, 5-11-13 Sugano, Ichikawa, Chiba 272-8513, Japan

E-mail: kuriharak@tdc.ac.jp

Key words: tongue squamous cell carcinoma, BMI1,ZEB1, epithelialmesenchymal transition
HNSCC patients, investigations into the underlying molecular and phenotypic events associated with head and neck squamous tumorigenesis are necessary. The identification of biomarkers for early detection and prognostic stratifications is also needed.

Recent findings have suggested that the persistent survival of cancer stem cells (CSCs), also known as tumor-initiating cells, may contribute to the aggression and recurrence of HNSCC (4-7). These CSCs are key contributors to radio- and chemoresistance and are responsible for tumor progression and recurrence after conventional therapy $(4,8)$.

Additionally, epithelial-mesenchymal transition (EMT) is a key developmental program that is often activated in CSCs during cancer development $(9,10)$. The occurrence of EMT in cancer cells may lead to a number of changes including loss of cell polarity and downregulation of epithelial cell markers, loss of cell-cell connections, in addition to gaining mesenchymal phenotypes along with genetic/epigenetic modifications of different genes. Published studies suggest a direct link between EMT and the gain of CSC-like properties (11). This process is thought to be a critical step in the induction of tumor metastasis and malignancy (12).

B-lymphoma Moloney murine leukemia virus insertion region-1 (BMI1), a member of the polycomb group (PcG) genes, is considered to be pivotal in regulating stemness-related genes involved in maintaining the self-renewal ability of stem cells by promoting chromatin modifications. BMI1 is also known to be deregulated in various human types of cancer (13-16). BMI1 is a prognostic marker in prostate, breast, ovarian, cervical, colorectal, lung, esophageal, gastric and nasopharyngeal cancer $(13,17-24)$. However, the role of BMI1 in maintaining self-renewal and tumorigenicity in HNSCC or HNSCC-derived cancer stem cells (CSCs) remains to be clarified.

ZEB1, a member of the zinc-finger transcription factor family, is one of the master regulators of EMT that mediates invasiveness as well as metastasis in many different types of malignant tumors. ZEB1 induces EMT by suppressing the expression of E-cadherin and contributing to the progression of malignant cancer (25). ZEB1 is a good predictor of prognosis in breast, lung, colorectal and esophageal cancer (26-29). Extensive studies have revealed that several transcription 
factors such as ZEB1 function together to regulate the EMT program (30). However, the role of ZEB1 in HNSCC remains unclear.

In the present study, we studied several biomarkers, BMI1, ZEB1, vimentin and E-cadherin associated with EMT in tongue squamous cell carcinoma (TSCC) cells and tumor specimens to determine their relationship to the invasion and progression of these tumors. TSCC accounts for $\sim 60 \%$ of oral squamous cell carcinoma and clarification of the significance of BMI1 and ZEB1 in this disease is critical for future therapies.

\section{Materials and methods}

Carcinoma cell lines and isolation of fibroblasts. Two human TSCC cell lines, Tosca-2S and Tosca-23, and human fibroblasts (31) were used. Human fibroblasts were collected from human oral specimens; tissues were cultured and the migrating fibroblasts were subcultured for 3-10 passages and used as stromal cells for this assay.

Collagen gel invasion assay. To conduct the collagen gel invasion assay, we used a 3-dimensional collagen gel culture. Insert chambers with $8-\mu \mathrm{m}$ pores were treated and placed in six $35-\mathrm{mm}$ culture dishes.

First, a collagen solution was poured into the chambers and incubated at $37^{\circ} \mathrm{C}$ for $30 \mathrm{~min}$ to solidify the gel. Second, eight volumes of acid-soluble $0.3 \%$ type I collagen solution (Cellmatrix type I-A, pH 3.0), one volume of $\mathrm{x} 10$ concentrated minimum essential medium, and one volume of reconstruction buffer ( $2.2 \mathrm{~g}$ of sodium bicarbonate and $4.77 \mathrm{~g}$ of HEPES dissolved in $100 \mathrm{ml}$ of $0.05 \mathrm{~N}$ sodium hydroxide) were mixed. Fibroblasts were added to this solution at a density of $1 \times 10^{5} / \mathrm{ml}$, subsequently, $2 \mathrm{ml}$ of this mixture containing fibroblasts was added to the chamber on top of the solid collagen-only layer. After solidifying, medium was added to the upper and lower parts of the well. TSCC cells were then spread on the gel.

The $35-\mathrm{mm}$ plates were observed using a phase-contrast microscope on a daily basis. Four weeks later, the whole collagen gel was fixed with $10 \%$ formalin, embedded in paraffin, cut into vertical $4-\mu \mathrm{m}$ sections, deparaffinized and stained with hematoxylin and eosin. For immunostaining, antigens were retrieved by heating at $120^{\circ} \mathrm{C}$ for $20 \mathrm{~min}$, and cancer cells were identified with vimentin, E-cadherin, BMI1 and ZEB1 antibodies.

The linear borderline between the cells and the gel corresponded to the basement membrane. The TSCC cells in the stratified layer along the gel or in contact with the basement membrane were considered preinvasive, and any downgrowth into the gel as invasion.

Tissue samples and patients. Tongue tissue specimens were accessed at the Oral Pathology of Showa University from 1997 to 2011, and were used in the present study. A total of 47 patients were eligible for inclusion ( 24 men and 23 women, with a median age of 58 years, range $30-83$ years). All the patients had undergone resection of the tongue primary tumor and did not include any patients with distant metastasis or any who had received preoperative therapy.

The present study was approved by the Ethics Committee, Oral Pathology of Showa University, and adhered to the principles in the Declaration of Helsinki. Samples were obtained after the patients had provided informed consent (permit no. 8, November 2, 2001).

Tongue tissues were surgically resected from the patients and hematoxylin and eosin-stained slides were assessed. The tissues were frozen in isopentane cooled in liquid nitrogen and stored at $-80^{\circ} \mathrm{C}$ for immunohistochemistry and RT-PCR. Sixty-four fresh-frozen samples were collected, of which 32 were primary invasive tongue cancers (15 early invasive that did not show any invasion into the muscle layer, and 17 advanced invasive cancers that had invaded the muscle layer) and 32 dysplasias (14 mild dysplasias and 18 moderate-severe dysplasias). Some cancer and dysplastic specimens were harvested simultaneously from one patient. The histological sections and immunostaining were analyzed by a single pathologist without knowledge of clinical data.

Immunohistochemistry for tongue tissues. The frozen tissues were cut into $4-\mu \mathrm{m}$ sections, fixed in $4 \%$ paraformaldehyde, and treated with $3 \%$ hydrogen peroxide in methanol for $10 \mathrm{~min}$ to block the endogenous peroxidase activity. Immunostaining was performed with a mouse monoclonal E-cadherin antibody (SC-8426, diluted 1:100 for IHC; Santa Cruz Biotechnology, Inc., Santa Cruz, CA, USA), or rabbit polyclonal BMI1 antibody (T3421, 1:100 for IHC; Cosmo Bio Epitomics, CA, USA), or rabbit polyclonal ZEB1 antibody (SC-25388, 1:100 for IHC; Santa Cruz Biotechnology, Inc.) overnight at $4^{\circ} \mathrm{C}$ or mouse monoclonal vimentin antibody (no. M0725; 1:200 for IHC; Dako, Glostrup, Denmark) for $30 \mathrm{~min}$ at room temperature. After rinsing in phosphate-buffered saline, the sections were incubated with biotinylated secondary antibody. Detection was performed with diaminobenzidine (DAB) and counterstained with Mayer hematoxylin followed by dehydration and mounting.

Assessment of immunohistochemical staining. Immunohistochemical staining was observed in the parabasal and basal cell layer of the normal squamous epithelium, the dysplasias, and the outermost layer of cancer cells at the invasive front. The semi-quantitative analysis of the stained sections was carried out by light-microscopy according to the immunoreactive scoring (IRS) system by Remmele and Stegner (32). Sections were examined at a magnification of $x 400$ and the staining intensity (SI) was assessed by comparison with adjacent normal epithelia, which served as a reference for moderate intensity (M). Tumor staining less intense than the basal layer of adjacent normal epithelia was classified as weak $(\mathrm{W})$, more intense staining as strong intensity (S), and no staining as negative $(\mathrm{N})$. When a tumor had different staining intensities we assessed 10 random areas and recorded the largest area of intensity among these 10 measurements as the intensity for that tumor. Based on the percentage of positive cells (PP), the samples were classified into five grades: grade 0 , $(0 \%)$; grade $1,(0-10 \%)$; grade $2,(11-50 \%)$; grade $3,(51-80 \%)$; and grade 4, (81-100\%). The product of SI and PP was the IRS. The IRS with points from 0 to 12 was adapted to an additional 3 -point IRS classification (Table I).

Laser microdissection. The $8 \mu \mathrm{m}$ sliced sample was fixed in 95\% ethanol for $5 \mathrm{~min}$, and then washed with $70 \%$ ethanol, and 
A

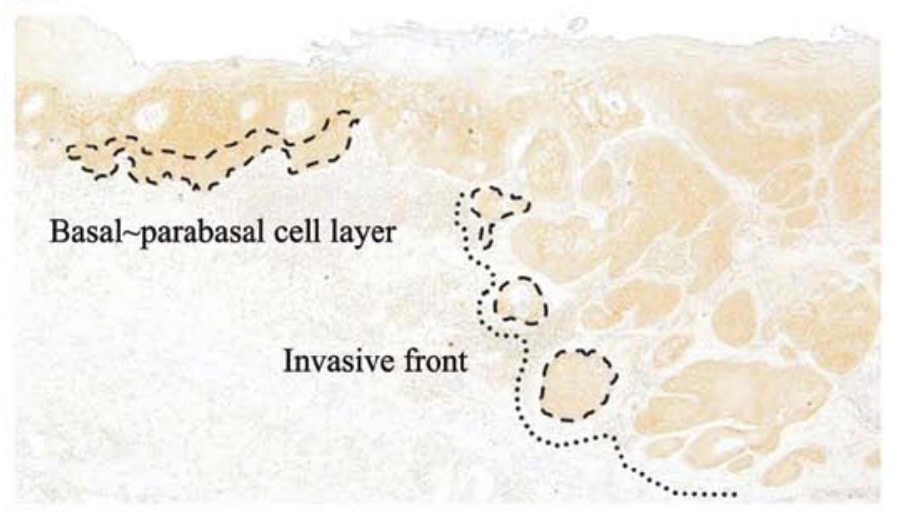

B

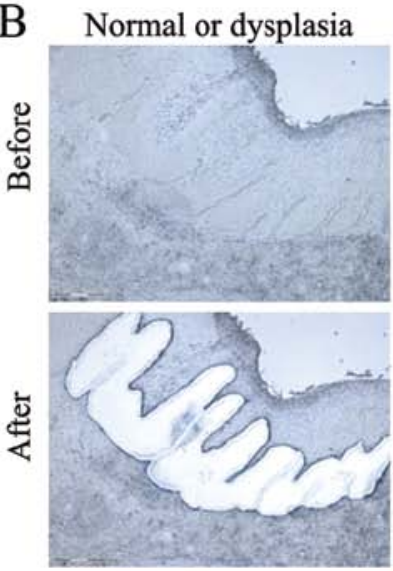

Carcinoma

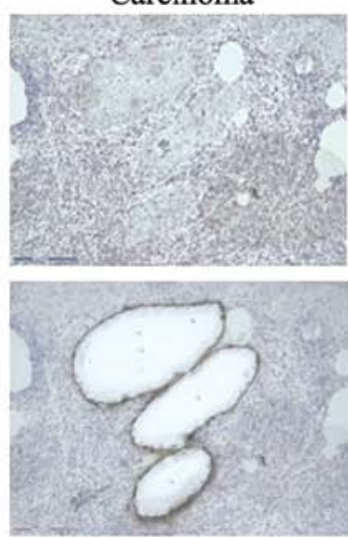

Figure 1. (A) Assessment of immunohistochemical staining and (B) specimen before and after laser microdissection. (A) Immunohistochemical protein expression levels were observed in the parabasal and basal cell layer of the normal squamous epithelium and dysplasia, in the invasive front of the invaded cancer. The semi-quantitative analysis of the stained sections was carried out by light microscopy according to the immunoreactive scoring (IRS) system by Remmele and Stegner (32). (B) mRNA expression levels as well as immunohistochemical protein expression were collected from the parabasal and basal cell layer of the normal squamous epithelium and dysplasia, in the invasive front of the invaded cancer. Total mRNA was independently extracted from each population of laser-microdissected cells.

Table I. Immunoreactive score (IRS) and IRS classification scoring systems.

\section{A, Immunoreactive score (IRS)}

Percentage of positive cells $\mathrm{x}$ intensity of staining, IRS (0-12)

0 , No positive cell

$1,<10 \%$ positive cells

$2,10-50 \%$ positive cells

$3,51-80 \%$ positive cells

$4,>80 \%$ positive cells

$\mathrm{B}$, IRS classification scoring systems

\begin{tabular}{lll}
\hline IRS-points & \multicolumn{2}{c}{ IRS-classification } \\
\hline $0-4$ & 0, Less than normal & Negative \\
$5-8$ & 1, Less than normal & Negative \\
$9-12$ & 2, More than normal & Positive \\
\hline
\end{tabular}

stained with the LCM frozen section staining kit (Ambion). We procured a few hundred cells from the cancers or dysplasias and the adjacent normal epithelia from 15 samples using laser microdissection (PALM MicroBeam; Zeiss, Boston, Massachusetts, USA) (Fig. 1A and B). The microdissected cells within the cap were covered with buffer and vortexed. Total RNA was extracted from each population of laser-microdissected cells.

Reverse transcription-quantitative PCR. Total RNA was extracted with the RNeasy Plus Micro kit (Qiagen, Valencia, CA, USA), mRNA was reverse transcribed with SuperScript VILO Master Mix (Invitrogen-Life Technologies, Carlsbad, CA, USA), and cDNA synthesis was performed. Quantitative
PCR was performed with an ABI PRISM 7500 Fast Real-Time PCR System (Applied Biosystems).

The amplification profile used was: denaturation at $95^{\circ} \mathrm{C}$ for $10 \mathrm{~min}$, followed by 50 cycles of denaturation at $95^{\circ} \mathrm{C}$ for $15 \mathrm{sec}$ and annealing at $60^{\circ} \mathrm{C}$ for $1 \mathrm{~min}$. The expression levels were quantified using the vimentin primer (Hs00185584_m1; TaqMan), E-cadherin primer (Hs01023894_m1; TaqMan), BMI1 primer (Hs00180411_m1; TaqMan), and ZEB1 primer (Hs00232783_m1, TaqMan) (all from Applied Biosystems). The geometric mean of the glyceraldehyde-3-phosphate dehydrogenase (GAPDH) was used as an internal control to normalize any variability. The comparative cycle threshold (CT) method was applied to quantify the relative expression levels of mRNAs. The relative amount of each marker was calculated using the equation $2^{-\Delta C_{T}}$ where $\Delta C_{T}=\left(C_{T X}-C_{T G A P D H}\right)$.

Statistical analysis. A comparison of the protein expression levels according to the IRS was carried out using the Kruskal-Wallis and the post hoc Mann-Whitney U tests. A comparison of mRNA expression levels was carried out using the non-repeated measures ANOVA and post-hoc Mann-Whitney U test. The correlation between the expression of several biomarkers in the TSCC cells was assessed with the Chi-square and Fisher's exact tests, and Spearman's correlation. Statistical analyses were performed using modified EZR (The R Foundation for Statistical Computing, Perugia, Italy) software programs. Two-tailed P-values were calculated and $\mathrm{P}<0.05$ and $<0.01$ were considered to indicate a statistically significant result.

\section{Results}

Protein expression in an in vitro TSCC invasion model. In our collagen gel invasion assay, we examined the association between protein expression and early invasion of TSCC cells (TOSCa-2S and TOSCa-23). Only $2.5 \%$ of preinvasive cells had a high vimentin expression, but as many as $70.0 \%$ of invasive cells had high vimentin levels. Seventy-two 


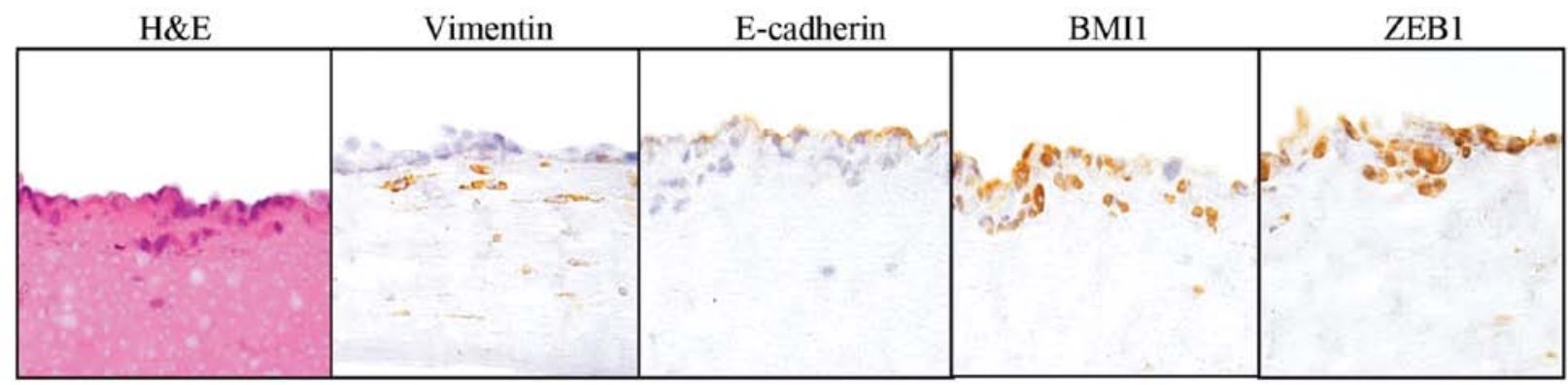

Figure 2. Protein expression of TSCC cells in collagen gel invasion assay. To conduct the collagen gel invasion assay, we used a 3-dimensional collagen gel culture. By the fourth week of cell culture, cancer cells were identified with vimentin, E-cadherin, BMI1 and ZEB1 (magnification, x400). TSCC, tongue squamous cell carcinoma; H\&E, hematoxylin and eosin staining.

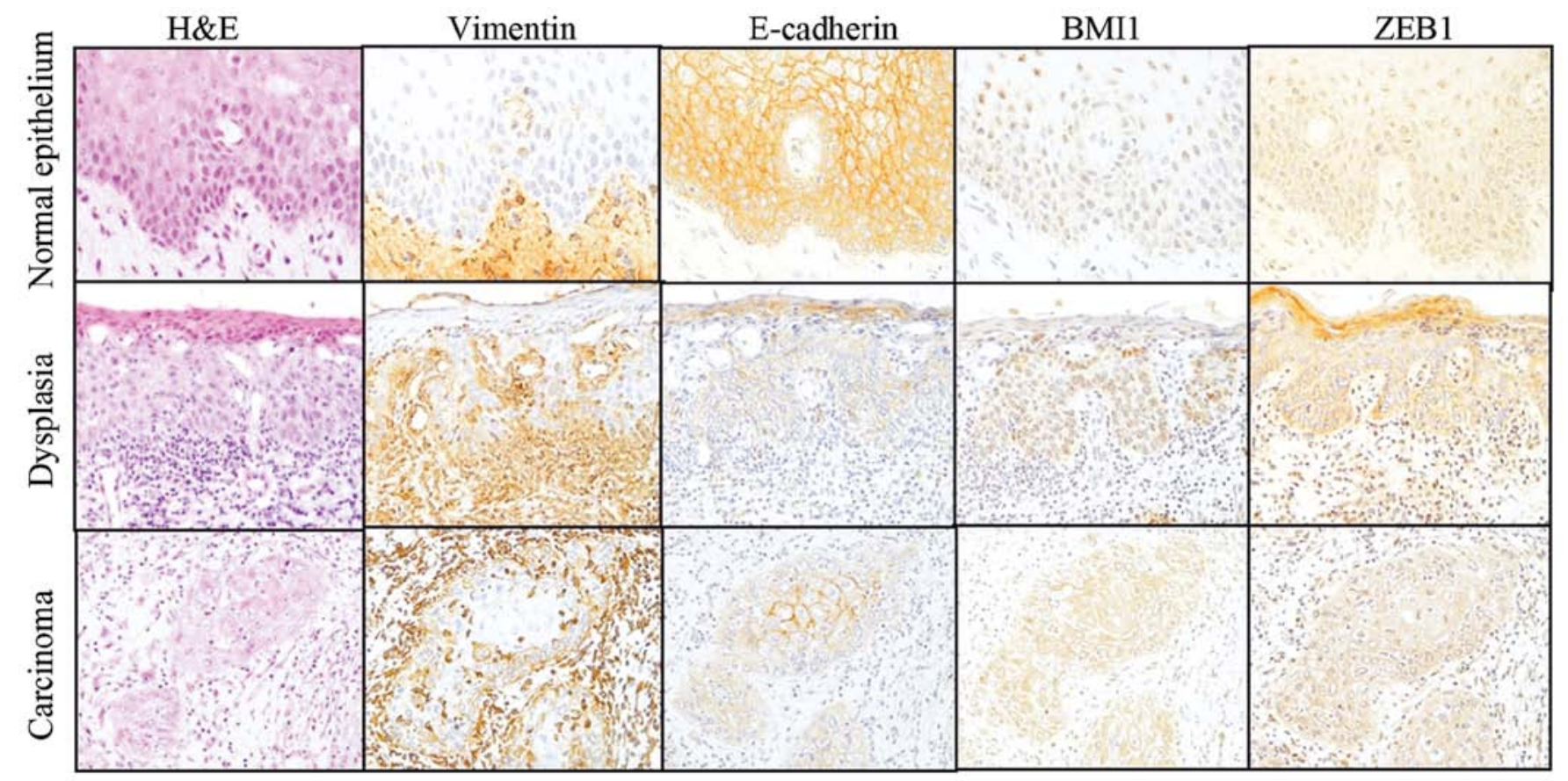

Figure 3. Immunohistochemical staining of tongue tissues. Immunohistochemical staining was performed with tongue tissue specimens showing the histological features of normal epithelium, moderate-severe dysplasia and invasive cancer. The representative examples of histology (H\&E, hematoxylin and eosin staining) and vimentin, E-cadherin, BMI1 and ZEB1 staining are shown (magnfication, x200).

percent of preinvasive cells and only $3.6 \%$ of invasive cells had high E-cadherin expression levels. Sixty-two percent of the preinvasive cells and $73.0 \%$ of the invasive cells showed a high BMI1 expression, and $60.2 \%$ of the preinvasive cells in addition to $73.5 \%$ of the invasive cells had high-ZEB1 levels (Fig. 2). Protein expression markers were significantly different between the preinvasive and invasive cells $(\mathrm{P}<0.001$, $\mathrm{P}<0.01$; Table II).

Protein and $m R N A$ expression in vivo using human tissue samples. We compared the expression of these proteins immunohistochemically among the five groups of samples: normal squamous epithelium, mild and moderate-severe dysplasia, early invasive and advanced invasive cancer. BMI1 immunoexpression was mainly detected within the nuclei of the normal squamous epithelium and mild dysplasia, but was detected in the nuclei and cytoplasm of the majority of severe dysplasia and cancer cells. BMI1 high immunoexpression (IRS-classification, 12) was observed in $84.4 \%(27 / 32)$ of the invasive cancers. ZEB1 expression was detected in the cytoplasm of the samples and ZEB1-high expression was only observed in $50.0 \%$ (16/32) of the invasive cancers (Fig. 3).

After scoring and assessing the immunohistochemical samples, E-cadherin expression was found to be significantly decreased in the moderate-severe dysplasia and invasive cancer samples compared with the adjacent normal squamous epithelium $(\mathrm{P}=0.0021, \mathrm{P}<0.001, \mathrm{P}<0.0001$; Fig. 4A). Vimentin and BMI1 protein expression levels were significantly increased in the invasive cancers, including early and advanced samples $(\mathrm{P}=0.0074, \mathrm{P}<0.001, \mathrm{P}=0.04,0.0001$; Fig. 4A). ZEB1 expression was significantly increased only in advanced samples $(\mathrm{P}=0.014$; Fig. 4A), compared with the adjacent normal squamous epithelia. Using quantitative PCR, E-cadherin mRNA levels were significantly decreased in moderate-severe dysplasias 
Table II. Protein expression analysis in the preinvasive and invasive cells.

\begin{tabular}{llrl}
\hline & \multicolumn{2}{c}{ Vimentin expression } & \\
\cline { 2 - 3 } Cells & Positive (\%) & Negative (\%) & P-value \\
\hline Preinvasive & $11(2.5)$ & $426(97.5)$ & $<0.001$ \\
Invasive & $79(70.0)$ & $34(30.0)$ & \\
\hline
\end{tabular}

\begin{tabular}{|c|c|c|c|}
\hline \multirow[b]{2}{*}{ Preinvasive } & \multicolumn{2}{|c|}{ E-cadherin expression } & \multirow{3}{*}{$<0.001$} \\
\hline & $334(72.3)$ & $128(27.7)$ & \\
\hline Invasive & $4(3.6)$ & $107(96.4)$ & \\
\hline
\end{tabular}

\begin{tabular}{|c|c|c|c|}
\hline \multirow{3}{*}{$\begin{array}{l}\text { Preinvasive } \\
\text { Invasive }\end{array}$} & \multicolumn{2}{|c|}{ BMI1 expression } & \multirow{3}{*}{$<0.01$} \\
\hline & $305(62.4)$ & $184(37.6)$ & \\
\hline & $135(73.0)$ & $50(27.0)$ & \\
\hline
\end{tabular}

ZEB1 expression

\begin{tabular}{|c|c|c|}
\hline Preinvasive & $240(60.2)$ & 159 (39.8) \\
\hline Invasive & $83(73.5)$ & $30(26.5)$ \\
\hline
\end{tabular}

Table III. Correlation of vimentin, E-cadherin and BMI1 mRNA expressions.

\begin{tabular}{|c|c|c|c|c|c|}
\hline \multirow[b]{3}{*}{ Expression } & \multirow[b]{3}{*}{ Grade } & \multicolumn{3}{|c|}{ BMI1 expression } & \multirow{3}{*}{ P-value } \\
\hline & & \multicolumn{2}{|c|}{$\mathrm{Neg}$} & \multirow{2}{*}{$\frac{\text { Pos }}{2}$} & \\
\hline & & 0 & 1 & & \\
\hline \multirow[t]{4}{*}{ E-cadherin } & Neg & 3 & 11 & 36 & 0.0097 \\
\hline & Pos & 3 & 8 & 6 & \\
\hline & & \multicolumn{3}{|c|}{ BMI1 expression } & \\
\hline & & & & Pos & P-value \\
\hline \multirow[t]{2}{*}{ Vimentin } & $\mathrm{Neg}$ & 5 & 14 & 22 & 0.035 \\
\hline & Pos & 1 & 5 & 20 & \\
\hline
\end{tabular}

${ }^{\mathrm{a} E s t i m a t e d ~ b y ~ C h i-s q u a r e ~ t e s t . ~ N e g, ~ n e g a t i v e ; ~ P o s, ~ p o s i t i v e . ~}$

and the invasive cancers compared with the adjacent normal squamous epithelia ( $\mathrm{P}=0.039, \mathrm{P}=0.019$; Fig. 4B). Vimentin, BMI1 and ZEB1 mRNA expression levels were significantly increased in the invasive samples compared with the adjacent normal squamous epithelia $(\mathrm{P}=0.047, \mathrm{P}=0.036, \mathrm{P}=0.045$; Fig. 4B).

Elevated levels of BMI1 were accompanied by the downregulation of E-cadherin and an upregulation of vimentin at the invasive front, demonstrating a significant negative correlation between BMI1 and E-cadherin protein and mRNA expression ( $\mathrm{P}=0.0097$; Table III, $\mathrm{P}=0.018$; Fig. 5). This result also suggested a significant positive correlation between BMI1 and vimentin protein and mRNA expression $(\mathrm{P}=0.035$; Table III, $\mathrm{P}=0.0008$; Fig. 5). There was also a significant positive correlation between ZEB1 and vimentin mRNA levels, as well as between BMI1 and ZEB1 mRNA ( $\mathrm{P}<0.001, \mathrm{P}=0.024$; Fig. 5).

\section{Discussion}

EMT is encountered in three distinct biological settings (33). The first setting is associated with implantation and embryonic gastrulation, which leads to the mesoderm and endoderm, as well as to the development and organization of several structures. Type 2 EMTs are engaged in the context of inflammation and fibrosis. These EMTs continue to occur overtime until infections are removed and the tissue is repaired. Type 3 EMTs occur in the context of tumor growth and cancer progression, when epithelia transform into cancer cells and undergo EMT, which enables invasion and metastasis. EMTs have E-cadherin transcriptional repression in common, and it is worth noting that E-cadherin loss is associated with the progression of papilloma to invasive carcinoma (33).

In the present study, we examined the involvement of BMI1 and ZEB1 in tongue carcinogenesis by comparing E-cadherin and vimentin expression levels in vitro and in vivo.

In the TSCC cell invasion assay, we demonstrated that downregulation of E-cadherin was observed in $96.4 \%$ of the invasive TSCC cells, suggesting that they underwent EMT. We revealed that the BMI1 and ZEB1 proteins were expressed in $73 \%$ of the invasive TSCC cells compared with $60 \%$ of the preinvasive TSCC cells. This finding indicates that higher levels of BMI1 and ZEB1 expression are associated with the EMT program and TSCC cell invasion.

In TSCC tissues, BMI1 protein and mRNA expression levels were significantly increased in the invasive cancer tissues including early and advanced samples compared with adjacent normal squamous epithelia. At the invasive front, elevated levels of BMI1 were accompanied by the downregulation of E-cadherin and the upregulation of vimentin. This result shows the significant negative correlation between BMI1 and E-cadherin expression, and the significant positive correlation between BMI1 and vimentin expression. These results suggest that BMI1 elevation at the mRNA and protein level is involved in the invasion and progression of TSCC. These are similar results with previous studies of other epithelial malignancies and support an important role for BMI1 activation in the downregulation of E-cadherin and the induction of EMT.

Other studies of these factors have found similar results. For example, Song et al demonstrated that Bmi-1 mRNA and protein expression levels were found to correlate with the invasion of nasopharyngeal carcinoma (13). Yang et al showed that BMI1 is essential for EMT during tumor development in head and neck cancer patients (14). Kang et al showed that Bmi-1 overexpression was observed in preneoplastic oral mucosal tissues, which included those with mild, moderate or severe epithelial dysplasia (34). The reason for the divergence from our results may be due to the different pathophysiology of oral squamous cell carcinomas (non-keratinizing vs. keratinizing). Furthermore, many of the former studies presented small series of patients $(\mathrm{N}=8,10)$ with oral dysplastic and carcinoma tissue, whereas our results included 64 cases. Notably, Häyry et al 

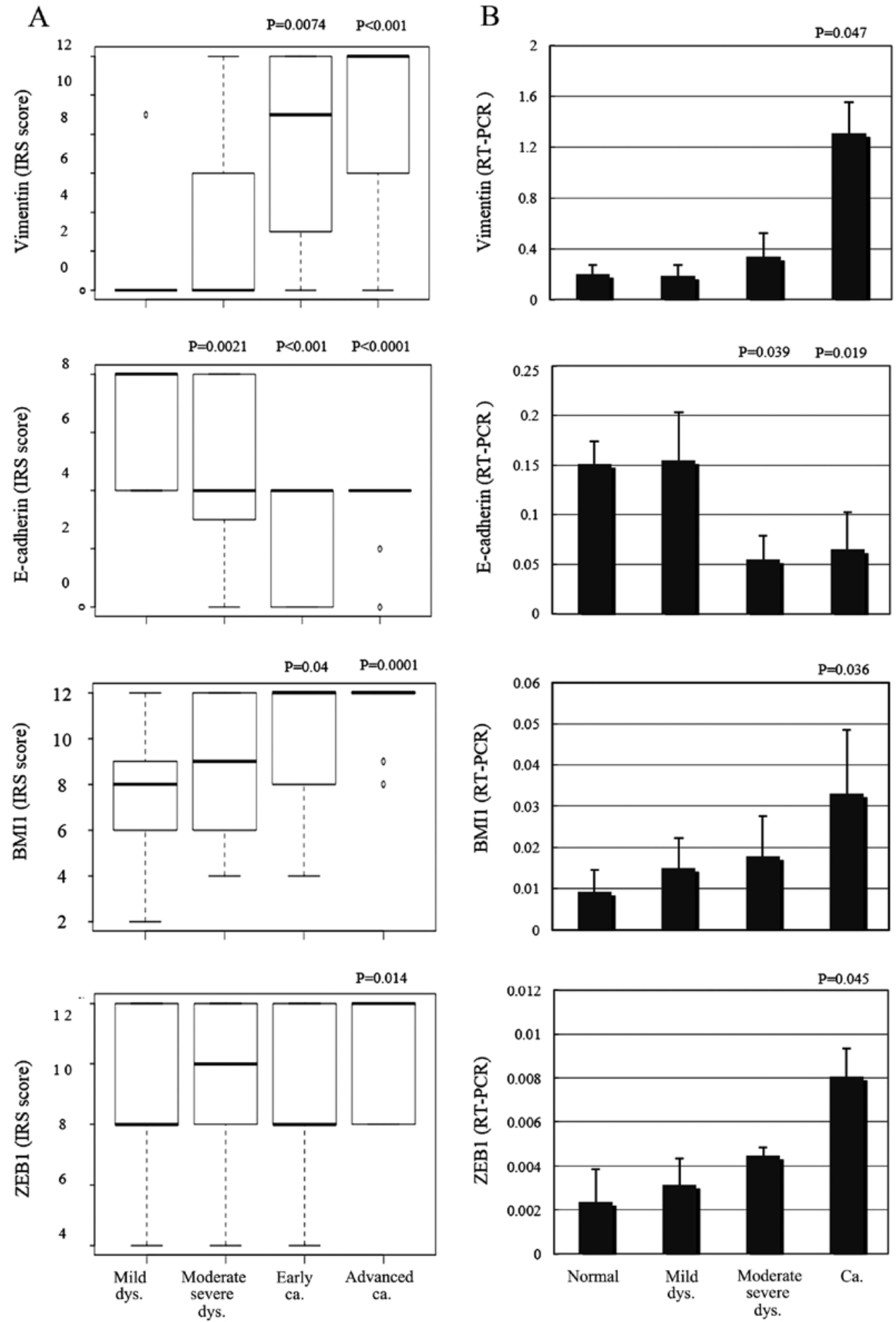

Figure 4. Vimentin, E-cadherin, BMI1 and ZEB1 protein expression according to the (A) IRS and (B) mRNA expressions. (A and B) Vimentin, BMI1 protein and mRNA expression levels were significantly increased in invasive cancer, ZEB1 expression was significantly increased in advanced invasive cancer compared with adjacent normal squamous epithelia. E-cadherin protein and mRNA expression levels were significantly decreased in moderate-severe dysplasia and invasive cancer compared with adjacent normal squamous epithelia. Dys, dysplasia; Ca, carcinoma; IRS, immunoreactive scoring.

showed a significant negative correlation between Bmi-1 protein expression and the recurrence of tongue cancer (35). This divergence from our results may be due to the samples used. Häyry et al used 1-mm biopsy punches that only provide information about focal points. Our samples provided a view of the entire tumor cross-section. Balasubramanian et al reported BMI1 expression in the basal and suprabasal keratinocytes but not in the surface epithelium (36). Recently, another study showed that in the invading front, BMI is highly enriched in CSCs, however, those authors found no Bmi-1 expression in 

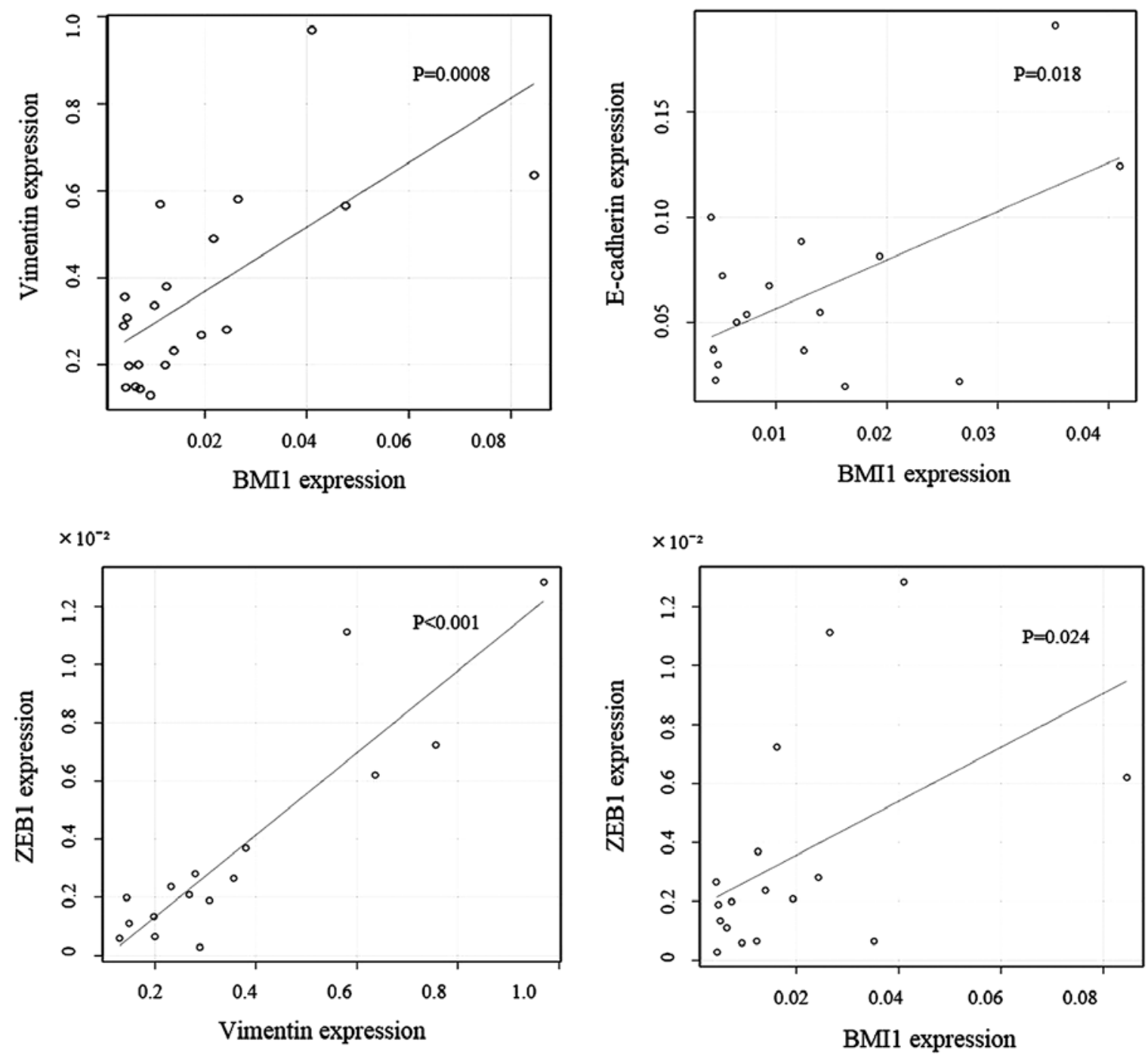

Figure 5. Correlation of the IHC grading of protein expression. A significant negative correlation between BMI1 and E-cadherin expression, and a significant positive correlation between BMI1 and vimentin expression is shown. There was a significant positive correlation between ZEB1 and vimentin mRNA expressions, between BMI1 and ZEB1 mRNA expressions.

the cancer cells (37). In the present study, we evaluated BMI1 protein and mRNA levels in cancer cells at the invasive front.

The overexpression of ZEB1 was observed in colorectal and esophageal cancer, suggesting an important role in tumorigenesis $(28,29)$. EMT-induced ZEB1 expression was also previously reported to be associated with cancer progression (38). We confirmed that ZEB1 protein expression was significantly increased in advanced invasive cancer compared with adjacent normal squamous epithelia, consistent with previous studies, and confirmed it to be associated with cancer progression.

ZEB1 was found to be responsible for the downregulation of basal membrane constituents at the invasive front in colorectal carcinoma (29), further, it has been shown that in esophageal SCC, ZEB1 downregulation by miR-150 suppressed E-cadherin repression, vimentin expression, migration ability and tumorigenicity (30). In the present study, we identified a significant positive correlation between ZEB1 and vimentin mRNA expression, but found no significant correlation between ZEB1 and E-cadherin expression. A hallmark of
EMT is the loss of E-cadherin expression; however, ZEB1 may not suppress E-cadherin itself. We observed a significant correlation between ZEB1 and BMI1 mRNA levels, which may indicate common regulation of BMI1 and ZEB1.

Recent fidings showed that EMT plays important roles in cancer invasion and metastasis by imparting cancer stem-cell properties $(4,9)$. One study demonstrated that ZEB1 and ZEB2 are key modulators of CSC properties in head and neck cancers, including EMT, metastasis and drug resistance (39). The results of the present study indicate that the activation of BMI1, a stem cell-like marker, is associated with the promotion of EMT and invasion in TSCC. Furthermore, elevated levels of BMI1 were accompanied by the downregulation of E-cadherin and upregulation of vimentin, and the elevated levels of ZEB1 were accompanied by the upregulation of vimentin at the invasive front of TSCCs.

We hypothesized that each of the variety of pathological tissues comprising oral cancers have individual roles in carcinogenesis. Further investigation is needed to elucidate the roles and mechanisms of BMI1 and ZEB1 in TSCC. In summary, 
our findings show that BMI1 and ZEB1 are important factors associated with the promotion of EMT and invasion of TSCC.

\section{Acknowledgements}

We thank the Showa University Pathology Department for technical assistance.

\section{References}

1. Haddad RI and Shin DM: Recent advances in head and neck cancer. N Engl J Med 359: 1143-1154, 2008.

2. Chen YJ, Chang JT, Liao CT, Wang HM, Yen TC, Chiu CC, $\mathrm{Lu} \mathrm{YC}, \mathrm{Li} \mathrm{HF}$ and Cheng AJ: Head and neck cancer in the betel quid chewing area: Recent advances in molecular carcinogenesis. Cancer Sci 99: 1507-1514, 2008.

3. Gibson MK and Forastiere AA: Reassessment of the role of induction chemotherapy for head and neck cancer. Lancet Oncol 7: 565-574, 2006.

4. Zhang Z, Filho MS and Nör JE: The biology of head and neck cancer stem cells. Oral Oncol 48: 1-9, 2012.

5. Rosen JM and Jordan CT: The increasing complexity of the cancer stem cell paradigm. Science 324: 1670-1673, 2009.

6. Lo WL, Yu CC, Chiou GY, Chen YW, Huang PI, Chien CS, Tseng LM, Chu PY, Lu KH, Chang KW, et al: MicroRNA-200c attenuates tumour growth and metastasis of presumptive head and neck squamous cell carcinoma stem cells. J Pathol 223: 482-495, 2011.

7. Chen C, Zimmermann M, Tinhofer I, Kaufmann AM and Albers AE: Epithelial-to-mesenchymal transition and cancer stem(-like) cells in head and neck squamous cell carcinoma. Cancer Lett 338: 47-56, 2013.

8. Major AG, Pitty LP and Farah CS: Cancer stem cell markers in head and neck squamous cell carcinoma. Stem Cells Int 2013: 319489, 2013.

9. Thiery JP, Acloque H, Huang RY and Nieto MA: Epithelialmesenchymal transitions in development and disease. Cell 139: 871-890, 2009.

10. Raimondi C, Gianni W, Cortesi E and Gazzaniga P: Cancer stem cells and epithelial-mesenchymal transition: Revisiting minimal residual disease. Curr Cancer Drug Targets 10: 496-508, 2010.

11. Polyak K and Weinberg RA: Transitions between epithelial and mesenchymal states: Acquisition of malignant and stem cell traits. Nat Rev Cancer 9: 265-273, 2009.

12. Thiery JP and Sleeman JP: Complex networks orchestrate epithelial-mesenchymal transitions. Nat Rev Mol Cell Biol 7: 131-142, 2006

13. Song LB, Li J, Liao WT, Feng Y, Yu CP, Hu LJ, Kong QL, Xu LH, Zhang X, Liu WL, et al: The polycomb group protein Bmi-1 represses the tumor suppressor PTEN and induces epithelialmesenchymal transition in human nasopharyngeal epithelial cells. J Clin Invest 119: 3626-3636, 2009.

14. Yang MH, Hsu DS, Wang HW, Wang HJ, Lan HY, Yang WH, Huang CH, Kao SY, Tzeng CH, Tai SK, et al: Bmil is essential in Twist1-induced epithelial-mesenchymal transition. Nat Cell Biol 12: 982-992, 2010.

15. Gil J, Bernard D and Peters G: Role of polycomb group proteins in stem cell self-renewal and cancer. DNA Cell Biol 24: 117-125, 2005.

16. Pasini D, Bracken AP and Helin K: Polycomb group proteins in cell cycle progression and cancer. Cell Cycle 3: 396-400, 2004.

17. Crea F, Duhagon Serrat MA, Hurt EM, Thomas SB, Danesi R and Farrar WL: BMI1 silencing enhances docetaxel activity and impairs antioxidant response in prostate cancer. Int J Cancer 128: 1946-1954, 2011

18. Guo BH, Feng Y, Zhang R, Xu LH, Li MZ, Kung HF, Song LB and Zeng MS: Bmi-1 promotes invasion and metastasis, and its elevated expression is correlated with an advanced stage of breast cancer. Mol Cancer 10: 10, 2011.

19. Wang E, Bhattacharyya S, Szabolcs A, Rodriguez-Aguayo C, Jennings NB, Lopez-Berestein G, Mukherjee P, Sood AK and Bhattacharya R: Enhancing chemotherapy response with Bmi-1 silencing in ovarian cancer. PLoS One 6: e17918, 2011.
20. Tong YQ, Liu B, Zheng HY, He YJ, Gu J, Li F and Li Y: BMI-1 autoantibody as a new potential biomarker for cervical carcinoma. PLoS One 6: e27804, 2011.

21. Kim JH, Yoon SY, Kim CN, Joo JH, Moon SK, Choe IS Choe YK and Kim JW: The Bmi-1 oncoprotein is overexpressed in human colorectal cancer and correlates with the reduced p16INK4a/p14ARF proteins. Cancer Lett 203: 217-224, 2004.

22. Vonlanthen S, Heighway J, Altermatt HJ, Gugger M, Kappeler A, Borner MM, van Lohuizen M and Betticher DC: The bmi-1 oncoprotein is differentially expressed in non-small cell lung cancer and correlates with INK4A-ARF locus expression. Br J Cancer 84: 1372-1376, 2001.

23. He XT, Cao XF, Ji L, Zhu B, Lv J, Wang DD, Lu PH and Cui HG: Association between Bmil and clinicopathological status of esophageal squamous cell carcinoma. World J Gastroenterol 15: 2389-2394, 2009.

24. Liu JH, Song LB, Zhang X, Guo BH, Feng Y, Li XX, Liao WT, Zeng MS and Huang $\mathrm{KH}$ : Bmi-1 expression predicts prognosis for patients with gastric carcinoma. J Surg Oncol 97: 267-272, 2008.

25. Kalluri R and Weinberg RA: The basics of epithelial-mesenchymal transition. J Clin Invest 119: 1420-1428, 2009.

26. Soini Y, Tuhkanen H, Sironen R, Virtanen I, Kataja V, Auvinen P, Mannermaa A and Kosma VM: Transcription factors zeb1, twist and snail in breast carcinoma. BMC Cancer 11: 73, 2011.

27. Gemmill RM, Roche J, Potiron VA, Nasarre P, Mitas M, Coldren CD, Helfrich BA, Garrett-Mayer E, Bunn PA and Drabkin HA: ZEB1-responsive genes in non-small cell lung cancer. Cancer Lett 300: 66-78, 2011.

28. Zhang GJ, Zhou T, Tian HP, Liu ZL and Xia SS: High expression of ZEB1 correlates with liver metastasis and poor prognosis in colorectal cancer. Oncol Lett 5: 564-568, 2013

29. Yokobori T, Suzuki S, Tanaka N, Inose T, Sohda M, Sano A, Sakai M, Nakajima M, Miyazaki T, Kato H, et al: MiR-150 is associated with poor prognosis in esophageal squamous cell carcinoma via targeting the EMT inducer ZEB1. Cancer Sci 104: 48-54, 2013.

30. Gheld of A, Hulpiau P, van Roy F, De Craene B and Berx G: Evolutionary functional analysis and molecular regulation of the ZEB transcription factors. Cell Mol Life Sci 69: 2527-2541, 2012.

31. Nakamura E, Kozaki K, Tsuda H, Suzuki E, Pimkhaokham A, Yamamoto G, Irie T, Tachikawa T, Amagasa T, Inazawa J, et al: Frequent silencing of a putative tumor suppressor gene melatonin receptor $1 \mathrm{~A}$ (MTNR1A) in oral squamous-cell carcinoma. Cancer Sci 99: 1390-1400, 2008.

32. Remmele W and Stegner HE: Recommendation for uniform definition of an immunoreactive score (IRS) for immunohistochemical estrogen receptor detection (ER-ICA) in breast cancer tissue. Pathologe 8: 138-140, 1987 (In German).

33. Nieto MA: The ins and outs of the epithelial to mesenchymal transition in health and disease. Annu Rev Cell Dev Biol 27: 347-376, 2011.

34. Kang MK, Kim RH, Kim SJ, Yip FK, Shin KH, Dimri GP, Christensen R, Han T and Park NH: Elevated Bmi-1 expression is associated with dysplastic cell transformation during oral carcinogenesis and is required for cancer cell replication and survival. Br J Cancer 96: 126-133, 2007.

35. Häyry V, Mäkinen LK, Atula T, Sariola H, Mäkitie A, Leivo I, Keski-Säntti H, Lundin J, Haglund C and Hagström J: Bmi-1 expression predicts prognosis in squamous cell carcinoma of the tongue. Br J Cancer 102: 892-897, 2010.

36. Balasubramanian S, Lee K, Adhikary G, Gopalakrishnan R, Rorke EA and Eckert RL: The Bmi-1 polycomb group gene in skin cancer: Regulation of function by (-)-epigallocatechin3-gallate. Nutr Rev 66 (Suppl 1): S65-S68, 2008.

37. Siddique HR and Saleem M: Role of BMI1, a stem cell factor, in cancer recurrence and chemoresistance: Preclinical and clinical evidences. Stem Cells 30: 372-378, 2012.

38. Chen ML, Liang LS and Wang XK: miR-200c inhibits invasion and migration in human colon cancer cells SW480/620 by targeting ZEB1. Clin Exp Metastasis 29: 457-469, 2012.

39. Chu PY, Hu FW, Yu CC, Tsai LL, Yu CH, Wu BC, Chen YW, Huang PI and Lo WL: Epithelial-mesenchymal transition transcription factor ZEB1/ZEB2 co-expression predicts poor prognosis and maintains tumor-initiating properties in head and neck cancer. Oral Oncol 49: 34-41, 2013. 